\title{
Hemorrhagic shock secondary to spontaneous rupture of a non- secretory adrenal cortical tumour: A case report
}

\author{
Omar A. Jarral, MRCS, ${ }^{*}$ Colin Todd, FRCR; ${ }^{\dagger}$ Peter D. Willson, FRCS ${ }^{\ddagger}$
}

\begin{abstract}
A 21-year-old female presented with left-sided loin pain and profound circulatory shock. After emergency laparotomy, angiography, embolisation and histological investigation, a diagnosis of spontaneous rupture of a benign non-secretory adrenal cortical tumour was made. To our knowledge, this is the only reported case of this diagnosis.
\end{abstract}

Can Urol Assoc J 2010;4(6):El61-E163

\section{Introduction}

In most clinical situations, the acute abdomen combined with profound shock and anemia in a young female with no history of trauma would lead to the consideration of a ruptured ectopic pregnancy or ovarian cyst. This case demonstrates an extremely rare cause of spontaneous retroperitoneal hemorrhage (a non-secretory benign adrenocortical tumour) in such a patient and highlights the need to consider all retroperitoneal organs in the management of hemorrhagic shock.

\section{Case presentation}

A 21-year-old previously well female presented to the emergency department with left-sided loin pain. Shortly after, she collapsed in the waiting area and was subsequently found to be profoundly hypotensive with a hemoglobin of $5.7 \mathrm{~g} / \mathrm{dL}$. On examination, a large left-sided abdominal mass was palpable. After general surgical and gynaecological review, it was decided to proceed immediately to laparotomy, with the shock presumed secondary to either the rupture of an ovarian cyst or an ectopic pregnancy. Radiological imaging was not obtained at this stage due to hemodynamic instability.

Laparotomy revealed a normal uterus and ovaries, and a significant retroperitoneal bleed which had ruptured to the peritoneal cavity. Bleeding was controlled with abdominal packs and the patient transferred to the intensive therapy unit (ITU). A re-look laparotomy the following day revealed further bleeding from abnormal veins around a ruptured left-sided retroperitoneal mass between the kidney and spleen which were oversewn. Re-look laparotomy at this stage rather than radiological imaging or intervention was chosen due to ongoing hemodynamic instability and the risks associated with transfer to an imaging suite. Surgical resection of retroperitoneal organs was not considered at this stage due to the uncertainty with regards to the source of bleeding. The patient required 30 units of blood.

Arterial angiography was performed on Day 2 (Fig. 1). This x-ray revealed a large bleeding mass in the left suprarenal area and an accessory renal artery which fed this mass with a large venous lake. Both of these supplying arteries were occluded with coils, which led to stabilization of the patient. A computed tomography (CT) scan of the abdomen/ pelvis demonstrated a resolving hematoma around the left suprarenal area (Fig. 2). No abdominal or pelvic lymph node enlargement was visible on the rest of the CT.

Biopsies demonstrated that among native tissue, there were fragments of an unencapsulated tumour, composed of nests and trabeculae of bland cells with round, occasionally enlarged and hyperchromatic nuclei, with a moder-

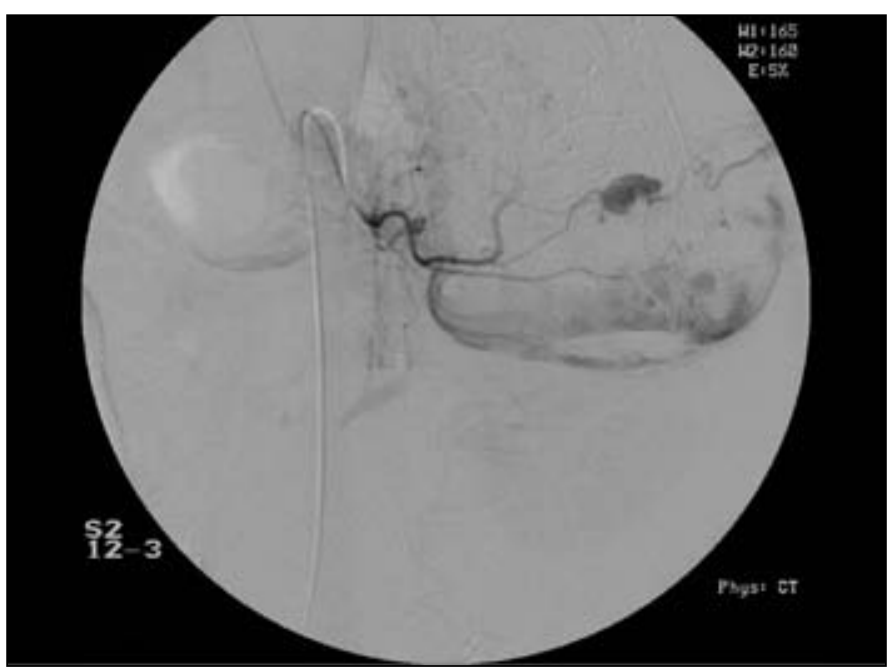

Fig. 1. Arterial angiography showing size of tumour and amount of bleeding. 


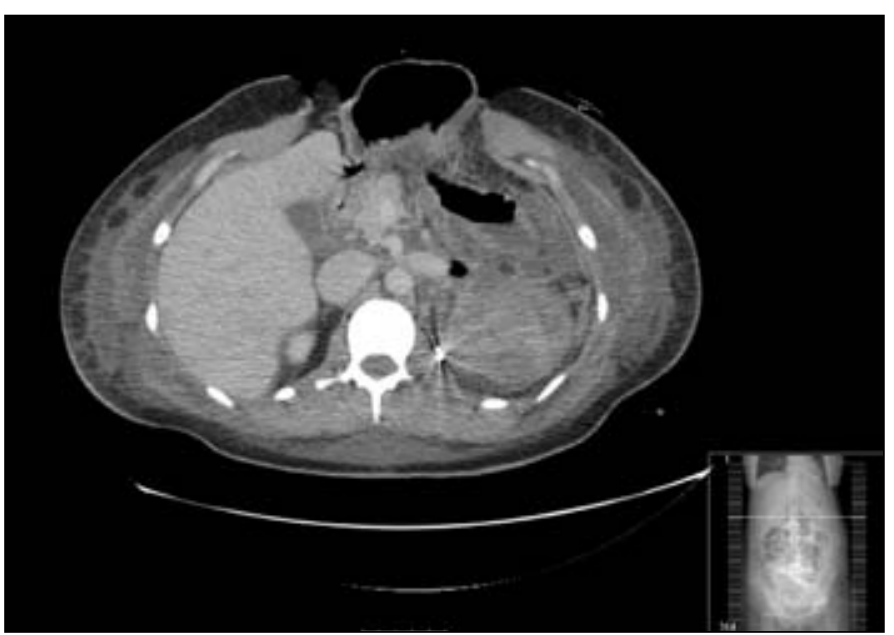

Fig. 2. A computed tomography of the abdomen/pelvis, showing a resolved suprarenal hematoma.

ate amount of granular cytoplasm. There was a significant amount of necrosis, a few mitoses (none of which atypical) and no tumour emboli were seen in the intralesional vessels (Fig. 3). Immunostains showed the tumour cells were negative for chromogranin, CK7, CK20, PGP9.5 serotonin, glucagon, somatostatin, gastrin and insulin (Fig. 4). There were no sustentacular cells with S100. They were positive for neuron-specific enolase, CD56, inhibin and Melan-A. Synaptophysin was equivocal. Vimentin and MNF appeared negative. In summary, the immunoprofile and histology were diagnostic of an adrenal cortical neoplasm with uncertain malignant potential. Short synacthen, dexamethasone suppression, 24-hour urinary catecholamines/cortisol, and renin activity were all within normal limits.

Treatment consisted of a combination of embolization of the offending vessels, multiple blood products (including Factor 7), laparostomy, regular re-look laparostomy and a

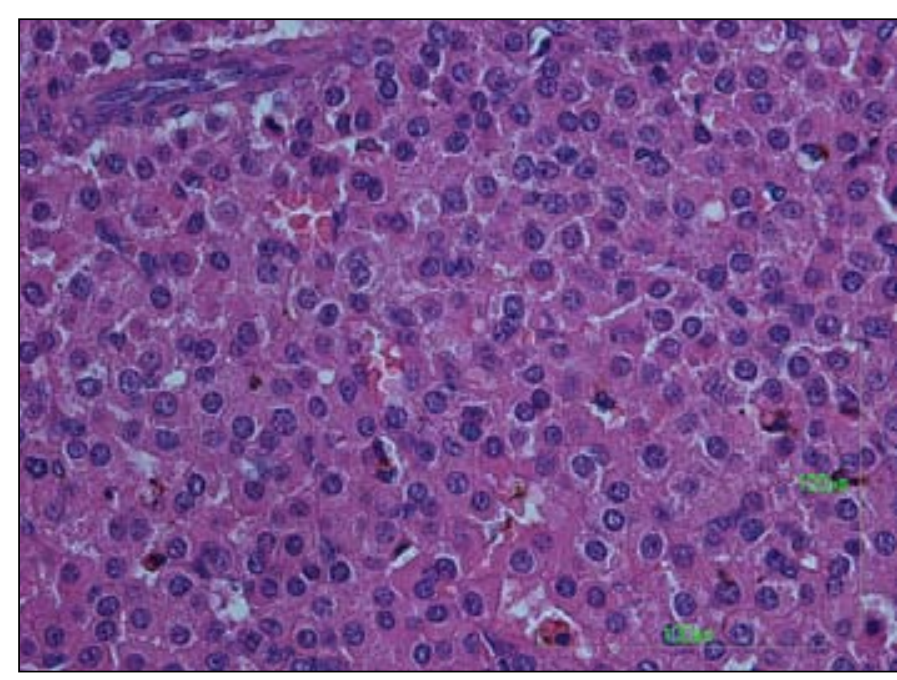

Fig. 4. High power view of tumour.

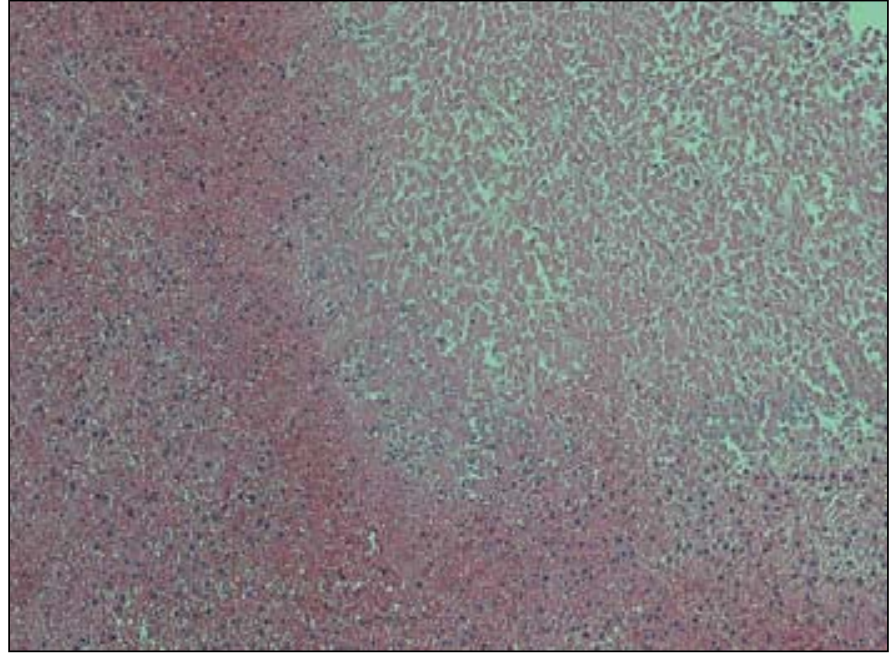

Fig. 3. Tumour necrosis visible on right side.

month in the ITU. The patient was discharged 8 weeks after admission.

\section{Discussion}

Spontanenous retroperitoneal hemorrhage is most commonly due to rupture of an aortic abdominal aneurysm and disorders of other retroperitonal organs, such as renal cell carcinoma. ${ }^{1}$ Adrenal tumours associated with spontaneous hemorrhage, such as phaeochromocytoma ${ }^{2,3}$ and adrenal cortical carcinoma, ${ }^{4}$ are particularly rare. The mention of benign tumours, such as myelolipomas, ${ }^{5}$ spontaneously rupturing are even more rare. To our knowledge, this case of a spontaneous hemorrhage of a benign adrenocortical tumour has not been mentioned in the literature.

\section{Conclusion}

Rarer causes of hemorrhagic shock must be considered early in patients with retroperitoneal bleeding. Benign adrenal tumours have the potential to bleed spontaneously in a castrosphic fashion. Arterial embolisation can be effective in both the diagnosis and management of such uncontrolled retroperitoneal hemorrhage and could have been considered earlier in this case despite hemodynamic compromise.

*Department of Biosurgery and Surgical Technology, Imperial College London, St. Mary's Hospital, London, UK; 'Department of Radiology, Kingston Hospital, Galsworthy Road, Surrey, UK; `Department of General Surgery, Kingston Hospital, Galsworthy Road, Surrey, UK.

Competing interests: None declared.

Acknowledgements: Dr. Graham Knee, Consultant Histopathologist, for interpreting and providing pathology images. 
This paper has been peer-reviewed.

\section{References}

1. Pode D, Caine M. Spontaneous retroperitoneal hemorrhage. J Urol 1992;147:31 1-8.

2. Hendrickson RJ, Katzman PJ, Queiroz R, et al. Management of massive retroperitoneal hemorrhage from an adrenal tumor. Endocr J 2001;48:691-6.

3. Kobayashi T, Iwai A, Takahashi R, et al. Spontaneous rupture of adrenal pheochromocytoma: review and analysis of prognostic factors. J Surg Oncol 2005;90:31-5.
4. Suyama K, Beppu T, Isiko T, et al. Spontaneous rupture of adrenocortical carcinoma. Am I Surg 2007;194:77-8.

5. Nakajo M, Onohara S, Shinmura K, et al. Embolization for spontaenous retroperitoneal hemorrhage from adrenal myelolipoma. Radiat Med 2003;21:214-9.

Correspondence: Dr. Omar A. Jarral, Department of Biosurgery and Surgical Technology, Imperial College London, St. Mary's Hospital, London W2 1NY; omarjarral@gmail.com 